This is a post-peer-review, pre-copyedit version of an article published in: Pop M., Wright G. (eds) Functional Imaging and Modelling of the Heart. FIMH 2017. Lecture Notes in Computer Science, vol 10263. Springer, Cham.

The final authenticated version is available online at: $10.1007 / 978-3-319-59448-$ $\underline{437}$

This version is subjected to Springer Nature terms for reuse that can be found at: https://www.springer.com/gp/openaccess/authors-rights/aam-terms-v1 


\title{
A Patient-Specific Computational Fluid Dynamics Model of the Left Atrium in Atrial Fibrillation: Development and Initial Evaluation.
}

\author{
Alessandro Masci ${ }^{1}$, Martino Alessandrini ${ }^{1}$, Davide Forti ${ }^{2}$, Filippo Menghini ${ }^{2}$, \\ Luca Dedé ${ }^{2}$, Corrado Tommasi ${ }^{3}$, Alfio Quarteroni ${ }^{2}$, Cristiana Corsi $^{1}$ \\ 1 Department of Electrical, Electronic and Information Engineering, \\ University of Bologna, Bologna, Italy. \\ 2 Chair of Modelling and Scientific Computing, Swiss Federal Institute of Technology \\ (EPFL), Lausanne, Switzerland. \\ ${ }^{3}$ Hospital "S. Maria delle Croci", Ravenna, Italy
}

\begin{abstract}
Atrial fibrillation (AF) is associated to a five-fold increase in the risk of stroke and AF strokes are especially severe. Stroke risk is connected to several AF related morphological and functional remodeling mechanisms which favor blood stasis and clot formation inside the left atrium. The goal of this study was therefore to develop a patientspecific computational fluid dynamics model of the left atrium which could quantify the hemodynamic implications of atrial fibrillation on a patient-specific basis. Hereto, dynamic patient-specific CT imaging was used to derive the 3D anatomical model of the left atrium by applying a specifically designed image segmentation algorithm. The computational model consisted in a fluid governed by the incompressible Navier-Stokes equations written in the Arbitrary Lagrangian Eulerian (ALE) frame of reference. In this paper, we present the developed model as well as its application to two AF patients. These initial results confirmed that morphological and functional remodeling processes associated to AF effectively reduce blood washout in the left atrium, thereby increasing the risk of clot formation. Our analysis is a step forward towards improved patient-specific stroke risk stratification and therapy planning.
\end{abstract}

Keywords: atrial fibrillation, left atrial appendage, stroke, risk stratification, patient-specific analysis, computational fluid dynamics.

\section{Introduction}

Atrial fibrillation (AF) is the most common form of arrhythmia worldwide [?]. Its prevalence is expected at least to double in the next 50 years as the population ages [?]. AF is associated to a five-fold increase in the risk of stroke [?] and $\mathrm{AF}$ related strokes are more severe and likely to be fatal [?]. Clinical scores used to stratify the risk of stroke, such as the recommended $\mathrm{CHA}_{2} \mathrm{DS}_{2}$-VASc score [?], are based on very generic empirical factors such as age, hypertension and diabetes mellitus. As a result, their predictive power remains low [?]. 
In this context, several clinical studies suggested that stroke risk stratification could be improved by using hemodynamic information on the left atrium (LA) and left atrial appendage (LAA) [?, ?]. These studies are based on the consideration that AF effectively alters the intra-atrial blood flow dynamics in such a way to yield blood stasis and, therefore, clot formation and embolism. One factor is anatomical remodeling, which consists in the progressive LA enlargement and increase in the LAA elongation [?]. A second factor is given by the altered mechanical function. Atrial contraction becomes completely chaotic and therefore ineffective during the AF episodes. Moreover, there is a progressive loss of atrial function even in sinus rhythm [?].

Yet, the exact way these complex mechanisms interplay cannot be assessed experimentally and remains largely unknown. In this respect, computational fluid dynamics (CFD) represents a unique tool to test different boundary conditions on a complex fluid dynamics system, such as intra-cardiac hemodynamics, in a non-invasive fully controllable and reproducible way. The aim of this study was therefore to develop a patient-specific CFD model of the LA in AF which could help elucidate the role of the key anatomical and functional features of AF on the LA blood flow. By that, the provided tool might be used for improving personalized stroke risk stratification and therapy planning.

Related work: Despite the relevance of the problem, the literature on CFD LA modeling in AF is very scarce [?, ?]. Due to the higher priority, the majority of modeling efforts focused ventricular fluid-dynamics. Moreover, existing atrial models consider almost exclusively electrophysiology. Few coupled atrioventricular CFD models exist but still with main focus on the ventricle [?].

The first CFD study of the LA in AF was the one by Zhang and coll. [?]. They performed a purely theoretical (i.e. not patient-specific) study making use of a simple toy model of LA anatomy and motion. Inflow boundary conditions were also accounted for by simplistic wave-forms. More recently, Koizumi [?] used a geometry segmented from a healthy volunteer. Hence, anatomical remodeling effects were not taken into account. A trivial motion model was used consisting of simple contraction/expansion in the direction from the mitral valve (MV) to the LA roof. Fixed pressure values taken from the literature were used as boundary conditions and the MV was simulated as an on-off switch.

Statement of the contribution: In this paper, we present what to our knowledge represents the first patient-specific model of atrial blood flow in AF. The main original contributions consist of: 1) LA anatomy was obtained from an AF patient, hence, anatomical remodeling was accounted for; 2) Atrial motion was extracted from patient data by image tracking; 3) We employed realistic inflow boundary conditions obtained from real Doppler measurements on AF patients; 4) we used a state-of-the art library for CFD simulations (LifeVt4) which has been extensively validated and optimized for fluid dynamics studies and cardiac applications [?].

\footnotetext{
${ }^{4}$ https://cmcsforge.epfl.ch/projects/lifev/
} 


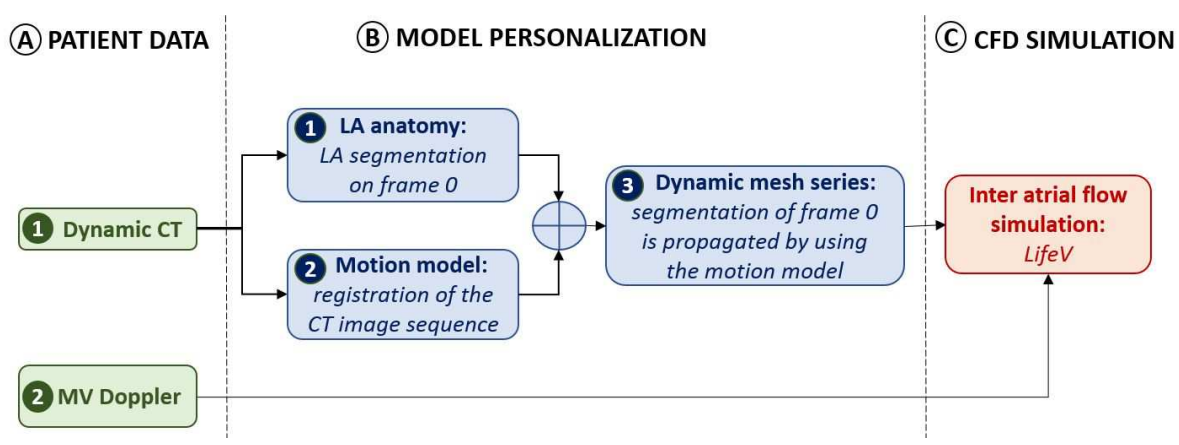

Fig. 1. Project flowchart.

In this paper, we present the development of our computational model as well as an initial evaluation on two AF patients, where the model is used to study the hemodynamic implications of an AF episode.

\section{Materials and Methods}

The project's flowchart is summarized in Fig. 1.

\subsection{Patient data for model personalization}

Clinical data was selected retrospectively from two AF patients. CT data (block A.1 in Fig. (1) was acquired from a Philips Brilliance 64 CT scanner. Ten volumes (170 axial slices, pixel size $0.4 \mathrm{~mm}$, slice thickness $1 \mathrm{~mm}$ ) spanning one cardiac cycle from the end of (ventricular) diastole were reconstructed by retrospective ECG gating.

Moreover, we used PW Doppler to set realistic inflow boundary conditions. Specifically, PW Doppler at the MV was used to constrain, indirectly, the inflow at the four pulmonary veins, as detailed in Sect. 2.3. Unfortunately, these meauserements were not available for the two considered patients. Representative MV velocity profiles were therefore taken from the literature [?] (block A.2 in Fig. (1).

\subsection{Patient specific anatomy and motion model}

Segmentation of the first frame - The 3D LA anatomy, inclusive of pulmonary veins (PV's) and left atrial appendage (LAA), was segmented from the first CT volume (block B.1 in Fig. (1). Each slice was processed independently, as follows.

For each slice, an initial segmentation was computed by intensity thresholding. A set of morphological opening and closing were then applied to remove 


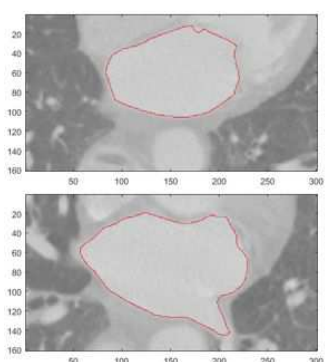

(a)

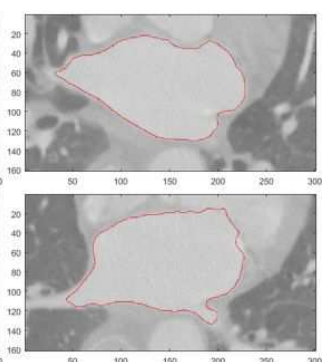

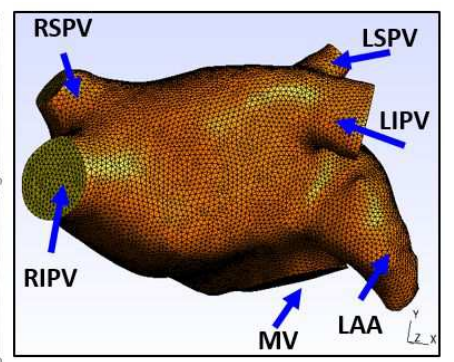

(b)

Fig. 2. Reconstructed anatomy for patient 1: (a) segmentation result on four slices; (b) final tetrahedral mesh. The principal anatomical structures are annotated in (b): right inferior PV (RIPV), right superior PV (RSPV), left inferior PV (LIPV), left superior PV (LSPV), LAA and MV. The final mesh contained 170,428 points and 1,042,766 tetrahedra.

spurious regions. Finally, a curvature based level set was used to regularize the contours [?].

Further processing was then required to make the 3D model compatible with the CFD simulator. This included smoothing via the HC Laplacian algorithm [?] and manual cutting of the inflow (i.e. the 4 PV's) and outflow (i.e. the MV) planes. Finally, a volumetric tetrahedral mesh was generated and the inflow/outflow surfaces were labeled with the VMTK library5. A final mesh quality check was performed. An example of segmentation in provided in Fig. 2 .

Motion model - We performed image registration on the full time sequence in order to extract the patient specific LA motion over the cardiac cycle (block B.2 in Fig. (1). Hereto, the displacement $\mathbf{d}_{i \rightarrow i+1}(\mathbf{x})$ between two successive CT volumes $I_{i}(\mathbf{x})$ and $I_{i+1}(\mathbf{x})$ was computed by elastic image registration [?], using the mean squared difference as a similarity metric.

The global displacement with respect to the reference volume at time 0 was then computed by accumulating the successive inter-frame estimates by means of the recursive formula $\mathbf{d}_{i \rightarrow 0}(\mathbf{x})=\mathbf{d}_{i \rightarrow i-1}(\mathbf{x}) \circ \mathbf{d}_{i-1 \rightarrow 0}(\mathbf{x})$, with $\mathbf{d}_{0 \rightarrow 0}(\mathbf{x})=\mathbf{0}$. To control error accumulation, the tracking was performed in the forward direction (i.e., with respect to frame 0 ) for half of the frames and in the backward direction (i.e., with respect to frame $N-1$, being $N$ the total number of frames) for the remaining half.

The displacement field was then used to propagate the initial mesh segmented at time 0 over the full cardiac cycle (block B.3 in Fig. 1). By calling $\mathbf{x}_{0}$ the position of a mesh node at time 0 , we computed its position $\mathbf{x}_{i}$ at time $i$ by sampling the computed displacement field, i.e. $\mathbf{x}_{i}=\mathbf{x}_{0}+\mathbf{d}_{i \rightarrow 0}\left(\mathbf{x}_{0}\right)$. As such, a set of $N$ tetrahedral meshes representing the instantaneous position of the LA on each available CT volume were obtained. Image registration and the sampling

\footnotetext{
${ }^{5}$ http://www.vmtk.org/
} 
of the diplacement field were all performed in Elastix [?]. All parameters were tuned to optimize tracking quality, as assessed visually.

Temporal interpolation - A continuous interpolation of the discrete mesh node positions was used to increase temporal resolution. Hereto, Fourier series interpolation was used, in agreement with the physiological periodicity of the heart motion. From a computational perspective, the continuous representation allowed to sample the model at a proper time step to ensure stability of the CFD solver. Moreover, thanks to the periodicity, an arbitrary number of cardiac cycles could be simulated, which was necessary in order to remove influence from the unphysiological initial condition on the fluid velocity.

\subsection{The computational model}

The model consisted in a fluid governed by the incompressible Navier-Stokes equations written in the Arbitrary Lagrangian Eulerian (ALE) frame of reference [?]. This conveniently splits the problem into two coupled sub-problems, namely the fluid problem, describing the fluid-dynamics, and the geometry problem, which attains to the motion of the computational domain. The latter determines the displacement of the fluid domain which, in turn, defines the ALE map.

For spatial discretization of the model, we consider a Galerkin finite element approximation using $\mathbb{P} 1-\mathbb{P} 1$ Lagrange polynomials with a suitable VMS-SUPG stabilization scheme [?,?] while for temporal discretization, we applied a second order semi-implicit backward differentiation formula scheme [?,?].

Inflow boundary conditions at each PV were assigned as follows. First, we considered a representative $\mathrm{MV}$ flow rate $Q_{0}$. The latter was computed as as $Q_{O}=v_{M V} A_{M V}$, being $v_{M V}$ mean velocity profile $v_{M V}$ (cf. Sect. 2.1) and $A_{M V}$ the MV cross sectional area.

The flow rate at each $\mathrm{PV}$ was then obtained by mass balance: $Q_{1}^{p v}+Q_{2}^{p v}+$ $Q_{3}^{p v}+Q_{4}^{p v}+Q^{O}+Q_{w a l l}=0$, where $Q_{i}^{p v}, i=\{1,2,3,4\}$ are the flow rates at each $\mathrm{PV}$ and $Q^{O}$ is the desired MV flow rate. $Q_{\text {wall }}$ is the flux associated to the LA volume variation, i.e. $Q_{\text {wall }}=d V / d t$, being $V$ the instantaneous LA volume.

Having defined $Q_{t o t}^{p v}=Q_{1}^{p v}+Q_{2}^{p v}+Q_{3}^{p v}+Q_{4}^{p v}$, the individual PV flow rates were then computed as: $Q_{l}^{p v}=\left(A_{l} / A_{t}\right) Q_{t o t}^{p v}-Q_{l}^{w}$, where $A_{l}$ is the sectional area and $A_{t}$ is the sum of all PVs sectional areas. $Q_{l}^{w}$ represents the flow due to the mesh velocity for each PV considering that the PVs sections move and their area changes during the cardiac cycle. Moreover, we chose to impose a parabolic velocity profile at each PV. Finally, we applied natural boundary conditions to penalize backflow at the MV [?]. The CFD simulations were performed through the LifeV library ${ }^{1}$ (block $\mathrm{C}$ of Fig. 11) in a parallel setting.

\subsection{Numerical Simulations}

We ran two patient-specific simulations from two sets of patient data. Each simulation was run for three cardiac cycles to remove influence from the unphysical 

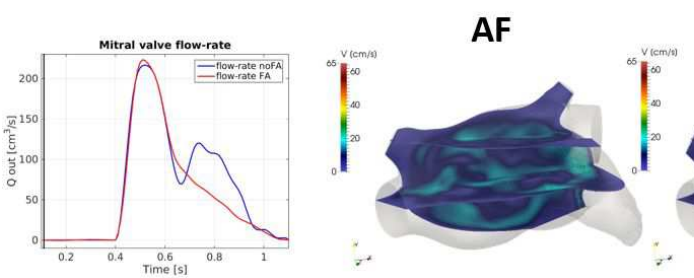

SR
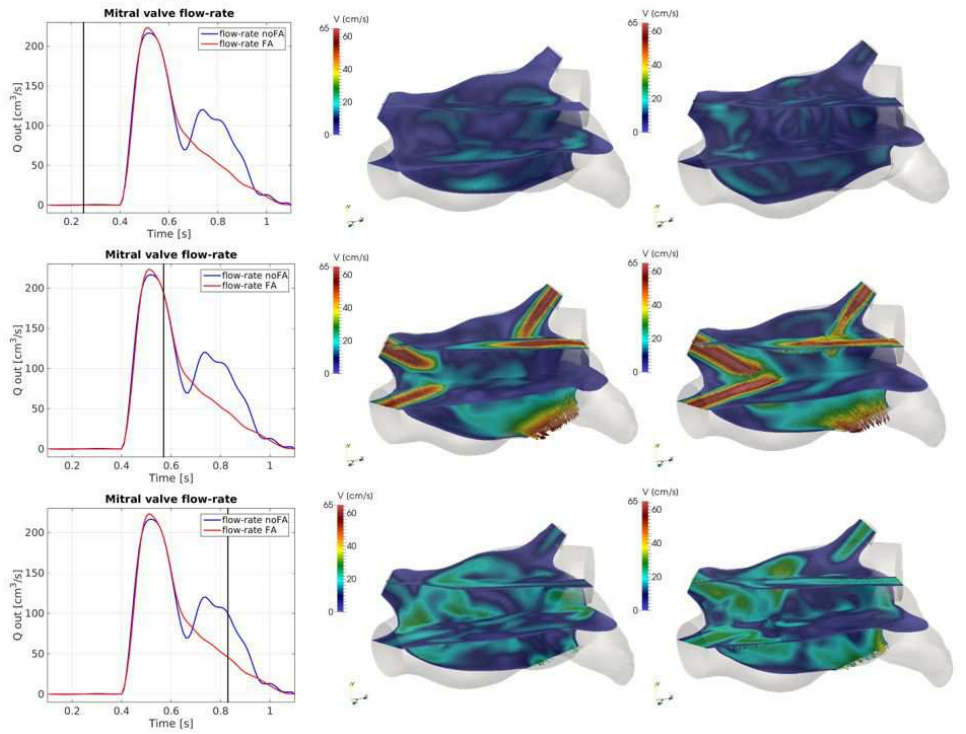

Fig. 3. Computed LA blood flow in one patient. First column reports the computed flow rate at the MV cut plane in SR (blue) and AF (red). Each row correspond to a different time in the cardiac cycle, denoted by the vertical black marker: end of diastole; LV sistole; LV filling (E-wave); atrial systole (A-wave). The computed flow velocity fields are reported in the second (AF) and third (SR) column.

initial conditions on the fluid velocities. The results on the 3rd simulated cycle will be therefore reported. For each patient, we run one simulation in sinus rhythm (SR) and one in AF. Hereto, SR employed the patient specific motion model extracted from the $\mathrm{CT}$ volumes, as described previously. AF was instead simulated by applying independently to each node a random displacement, that was kept small $(\approx 0.01 \mathrm{~mm})$ to avoid numerical issues arising from an excessive worsening of the mesh quality. Moreover, in AF, we redefined the inflow boundary conditions by considering a representative MV flow rate by removing the A-wave. 


\section{Results}

The simulated LA blood velocity is displayed in Fig. 3 for the first patient. We note that at end of diastole (first row) zero velocity is measured at MV, as expected, and PV's. During LV systole (second row) MV velocity is zero. However, we noticed an increment to $20 \mathrm{~cm} / \mathrm{s}$ at the four PVs $(20 \mathrm{~cm} / \mathrm{s})$ in SR due to atrial diastole. Due to the random motion model, this was not the case in AF, where PV velocities remained mostly null. During LV filling (third row), the MV flow increased (E-wave) to a peak value of about $65 / 70 \mathrm{~cm} / \mathrm{s}$ in both $\mathrm{SR}$ and AF simulations. An expected increase of the PV velocity was also confirmed by the simulation results (mean value $40 \mathrm{~cm} / \mathrm{s}$ ). During atrial systole (fourth row) we saw the expected A-wave at the MV (peak velocity between 35 and 40 $\mathrm{cm} / \mathrm{s}$ ) while the A-wave was missing in AF. Besides peak and mean values, the model allows to access the full complexity of the LA blood flow, cf. Fig. 3. In particular, detailed flow features, such as small vortexes, were represented by the model even if they are not discussed here due to the lack of space.

Blood stasis in the LAA might result in the formation of blood clots [?]. As a measure of blood stasis, we populated the LAA with 500 point particles, and counted how many remained inside the LAA after each cardiac cycle. The results for the two patients are reported in Tab. 1. After 3 cycles, $26 \%$ of the particles remained in the LAA in SR, while $45.6 \%$ remained in AF for patient 1. For patient 2, 39\% remained in SR and $50 \%$ in AF. In both cases, although more incisively for patient 1 , AF implied an expected reduced washout of the LAA which in the long term might be indicative of the generation of blood clots.

Table 1. Number of particles which remained in the LAA at the end of each cardiac cycle for the two considered patients in SR and AF. The percentage indicates the current number of particle with respect to the initial umber. Cardiac cycle $=0$ indicates the beginning of the simulation.

\begin{tabular}{c|cc|cc}
\hline & \multicolumn{2}{c}{ Patient 1 } & \multicolumn{2}{c}{ Patient 2 } \\
\hline Cardiac cycle & SR & AF & AF \\
\hline 0 & 500 & 500 & 500 & 500 \\
1 & 500 & 500 & 500 & 499 \\
2 & 478 & 480 & 455 & 465 \\
3 & $130(26 \%)$ & $228(45 \%)$ & $195(39 \%)$ & $251(50.2 \%)$ \\
\hline
\end{tabular}

\section{Conclusions}

We presented what to our knowledge represents the first patient-specific model of atrial hemodynamics in AF. An initial evaluation of the model was also presented to study the effect of an AF episode on the intra-atrial blood flow. 
In this initial testing, the model returned realistic blood flow patterns both at $\mathrm{SR}$ and during $\mathrm{AF}$. The model confirmed that $\mathrm{AF}$ episodes result in a reduced washout of the LAA which can lead to the formation of thrombi.

One limitation is that the model was not benchmarked against experimental flow measurements. Moreover, a comparison against a healthy atrium is necessary to understand the hemodynamic implications of AF better. Finally, an evaluation on more patients including a diverse range of anatomies and blood flow profiles has to be performed.

This is our initial effort towards the development of a better tool for stroke risk assessment and therapy delivery in the atrial fibrillation patient. 


\section{Acknowledgment}

This project has received funding from the European Union's Horizon 2020 research and innovation programme under the Marie SklodowskaCurie grant agreement No 659082. 